\title{
Gramática Espacial Urbana: la orientación espacial guiada por reglas verbales
}

\author{
Spatial and Urban Grammar: spatial orientation focuses on \\ verbal rules
}

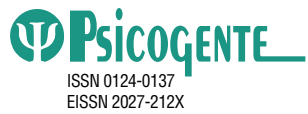

ARTÍCULO DE INVESTIGACIÓN Copyright (C) 2018 by Psicogente

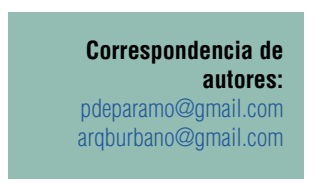

Recibido: 08-08-17 Aceptado: $18-06-18$ Publicado: 08-01-19

\section{Gramática Espacial Urbana: a orientação espacial guiada por} regras verbais

\author{
Pablo Páramo (iD - Andrea Burbano iD \\ Universidad Pedagógica Nacional, Bogotá, Colombia
}

Resumen

Objetivo: Analizar el papel que juegan las reglas en forma de instrucciones verbales y conducta no vocal sobre la manera como las personas se orientan en el espacio urbano.

Método: Investigación descriptiva de tipo exploratoria. Se seleccionaron de manera aleatoria 75 personas, hombres y mujeres, mayores de edad y de diversas condiciones sociales, a quienes se les indagó mediante entrevistas informales en distintos puntos del espacio público de Bogotá por la manera de llegar a un destino específico. La información fue recogida por asistentes de investigación quienes se hacían pasar por transeúntes en busca de un lugar mientras grababan la conversación con el entrevistado. Las entrevistas fueron analizadas mediante el programa Atlas.ti.

Resultados: Permiten agrupar las respuestas de los transeúntes a los lugares de destino en cinco categorías de reglas o instrucciones que dan las personas: instrucciones de secuencia geográfica, de secuencia de lugares, instrucciones para reconocimiento del lugar, instrucciones temporo-espaciales y no vocales.

Conclusiones: Las personas estructuran su conocimiento del espacio urbano a partir de un sistema de categorías verbales que tienen que ver con el seguimiento de reglas, las cuales se reflejan en instrucciones y comunicación no vocal o gestual a manera de expresiones corporales. Se discuten las implicaciones del estudio en la identificación de reglas que configuran una gramática espacial en contraposición a los modelos cognoscitivos y las aproximaciones de las neurociencias, así como los alcances para la planeación urbana y la enseñanza de la ubicación especial.

Palabras clave: Cognición espacial, Búsqueda de rutas, Conducta guiada por reglas, Orientación espacial.

Abstract

Objective: This research study aims to examine the role of verbal instructions and non-vocal behavior, in order to explore the way people are oriented in urban space.

Method: A descriptive and exploratory method was conducted. 75 adult people of different social strata, men and women, randomed selected were informaly interviewed about how to reach to a specific place, in different public places of Bogota. Research assistants who were in charge of data collecting, act as pedestrian recorded the conversation of the interviewee. Interviews were analyzed using Atlas.ti software.

Results: Showed five categories of rules or instructions given by people whom were interviewed in relation with destinations, including the interpretation that could be made on non-vocal expressions.

Conclusions: People structure their knowledge of urban space based on a system of verbal categories that have to do with the following of rules which are reflected in instructions and non-vocal or gestural communication in the form of corporal expressions. The implications of the study are discussed in the identification of rules that configure a spatial grammar, as opposed to cognitive models and neuroscience approaches, as well as the scope for urban planning and the teaching of special location.

Keywords: Spatial cognition, Wayfinding, Behavior focuses on rules, Spatial orientation.

Cómo citar este artículo (APA): Páramo, P. y Burbano, A. (2019). Gramática Espacial Urbana: la orientación espacial guiada por reglas verbales. Psicogente 22(41), 1-25. https://doi. org/10.17081/psico.22.41.3313 


\section{INTRODUCCIÓN}

Es bien sabido de las dificultades que tienen las personas para orientarse en la ciudad cuando tienen que ir a un lugar desconocido, hacer uso del transporte urbano y compartir espacios con extraños, lo que muchas veces produce estrés (Moser, 2012). Las grandes ciudades de hoy resultan confusas aun para quienes llevan años viviendo en ellas. La lectura de los mapas del transporte, por ejemplo, resulta incomprensible para muchos pasajeros (Bronzaft, Dobrow \& O'Hanlon, 1976). Resulta previsible suponer que en las ciudades las personas tampoco conocen o entienden las señales que se ubican de forma simbólica en los espacios públicos para orientarse por lo que tienen que recurrir a otros individuos para conseguir información (Levine, 1982). El problema de la ubicación es aún mayor para las personas mayores, quienes se pierden muchas veces al tratar de regresar a casa (Devlin, 2012). El presente trabajo tuvo como propósito identificar, a partir de las instrucciones o reglas verbales que da una persona para orientar a otra en el seguimiento de una ruta, la manera como se estructuran dichas reglas y si estas pueden configurar una gramática espacial que le permita al individuo posicionarse en el espacio.

\subsection{Gramática espacial urbana}

La gramática es considerada la rama de la lingüística que tiene por objeto el estudio de la estructura y el sistema que conforma un lenguaje: la escritura de las palabras (ortografía), de su forma y composición (morfo-fonología), de su significado (semántica), así como de su interrelación dentro de la oración o de la frase (sintaxis) (Diccionario Real Academia de la Lengua Castellana, 2014). En este trabajo se utiliza la gramática espacial a manera de metáfora con el propósito de atrapar los conceptos y estudios que se han realizado para entender de qué modo las personas exploran el espacio urbano, reconocen dónde están y se orientan para llegar a un lugar, a lo que se ha denominado encuentro de lugares o seguimiento de rutas, conceptos estos, con los que los investigadores se han aproximado al estudio de la conducta espacial o cognición espacial en lo que pareciera obedecer a una estructura similar a lo que sucede en la gramática.

Son varias las teorías propuestas hasta ahora para explicar la conducta espacial pero para efectos de su relevancia en el estudio que aquí se recoge, se hará mención brevemente a algunos de los argumentos de las teorías cognoscitiva y neurológica para presentar posteriormente una aproximación centrada en la regulación verbal que sustenta el presente estudio. 


\subsection{La aproximaciónes cognoscitivas y neurológicas}

La autolocalización y la capacidad de navegación han sido fundamentales para la supervivencia de las especies. La capacidad que tienen los organismo, según la teoría cognoscitiva de procesar información sensorial y guardar detalles de la topografía y geometría de un lugar, así como la de recordar una ruta que se ha tomado, ha permitido a las especies acceder a recursos para alimentarse e interactuar con otros individuos o grupos, y a veces esconderse. Los trabajos originales que dieron lugar a las primeras investigaciones sobre la orientación espacial provienen de estudios con animales del psicólogo Tolman, quien fue el pionero del constructo denominado mapas mentales (Tolman, 1948), los cuales han seguido desarrollándose en la investigación animal (Morris, 1981; Roberts, 1984; Hafting, Fyhn, Molden \& Moser, 2005; Kenneth, Lohmann, Lohmann \& Putman, 2007).

Por su parte, las investigaciones de O'Keefe (1976) sirvieron de soporte neurológico para la existencia de estos mapas mentales al identificar unas neuronas, a las que llamó células de posicionamiento, las que se comportaban como marcadores de localización al encenderse repetida y únicamente en algún lugar reconocido por el sujeto experimental, que para estos trabajos eran ratones de laboratorio. En 2005, May-Britt Moser y Edvard Moser, junto a O'Keefe descubrieron otro componente del sistema de posicionamiento del cerebro que los haría merecedores del Nobel en fisiología y medicina en 2014. Ellos encontraron un tipo de célula nerviosa a la que denominaron células de rejilla, las cuales generan un sistema de coordenadas que permite el posicionamiento preciso y la búsqueda de la ruta o la navegación y sirve para monitorear la posición del individuo en el ambiente. Este hallazgo ha resuelto, desde el punto de vista neurológico, el mecanismo mediante el cual el cerebro, al menos en ratones, crea un mapa del espacio que lo rodea, y cómo es que se facilita la navegación por un ambiente complejo. En el caso de los humanos, se sabe por ahora, que las memorias de eventos están relacionadas con las de los lugares donde sucedieron (Manzanero, 2008), lo cual hace suponer un mecanismo neurológico similar al identificado por O’Keefe, Moser y Moser.

La manera como se ha explorado la ubicación en el espacio en los humanos, en la actualidad, ha sido mediante la elaboración de dibujos, a los que también se denomina mapas cognoscitivos o mentales, los cuales se han propuesto como constructo hipotético para explicar las representaciones que las personas hacen del espacio y cómo se orientan para llegar a un lugar (Lynch, 1960; Stea, 1974; Navarro, Lozano \& Rodríguez, 2017). A finales de 
los años 50 del siglo pasado, Lynch pidió a sus participantes que hicieran un dibujo de las ciudades en que vivían con el propósito de explorar la imagen pública de Boston, Nueva Jersey y Los Ángeles, lo que le permitió identificar lo que para las personas es importante, o menos importante por omisión, en las representaciones que tiene de las ciudades, incluidos los hitos, bordes, sendas, nodos y barrios (Lynch, 1960). A partir de la identificación de estas propiedades de los mapas mentales el autor afirma que una buena ciudad debe ser legible, por lo cual entiende la facilidad con que sus partes pueden ser reconocidas y pueden organizarse dentro de un patrón coherente. Si bien Lynch no especifica cómo se establece esta coherencia, su estudio, constituye un importante precedente metodológico en la medida en que introduce el dibujo que hacen los individuos sobre sus ciudades para investigar la imagen que los habitantes tienen de estas y por consiguiente, su representación del espacio.

De este modo, el mapa cognoscitivo es visto como una representación mental que vincula la percepción y la toma de decisiones, lo que permite conocer en dónde se está, lo que puede seguir a continuación, y algunas pautas para la acción a seguir (Kaplan, 1973). Según Downs y Stea (2005), el mapa cognitivo se presenta como un constructo que abarca aquellos procesos que hacen posible a la gente adquirir, codificar, almacenar, recordar y manipular la información acerca de la naturaleza, de su ambiente espacial y es a la vez un recurso cognoscitivo del individuo para afrontar su ambiente cotidiano, a menudo sobrecargado de información (De Alba, 2004).

La exploración sobre estos mapas ha estado apoyada en estudios en los que se pide a los individuos: identificar una ciudad en un mapa (Matthews, 1987; Navarro, Lozano \& Rodríguez, 2017); listar elementos de un campus universitario (Holding, 1992), estimar distancias (Kirasic, Allen \& Siegel, 1984) y señalar un destino (Anooshian \& Young, 1981). También se han llevado a cabo varios trabajos que indagan por el modo como en diferentes culturas se construyen los mapas cognoscitivos (Kitchin \& Blades, 2002), o la incidencia de la variable género, investigacionesen las que se evidencian las diferentes estrategias que siguen hombres y mujeres para conseguir una orientación de ruta exitosa (Milgram \& Jodelet, 1976). Este tipo de trabajos se enmarcan dentro del campo que se le denomina cognición espacial (Devlin, 2001; 2012) al que se le suman la investigación sobre la búsqueda de ruta y los que provienen de la teoría de la comunicación, que incluyen la señalización y la comunicación (Pezzulo, Donnarumma \& Dindo, 2013). 
Por su parte, la aproximación neurocognoscitiva ha permitido identificar y entender, gracias a las tecnologías fMRIs (functional Magnetic Resonance Imaging) y escaneos PET (Positron Emission Tomography), varios procesos cerebrales para estudiar algunas partes del cerebro vinculadas con la búsqueda de rutas como el hemisferio derecho y en especial el hipocampus (Morrow \& Ratcliff, 1988), con lo que se podría pensar que al ubicar estas zonas y su actividad en la cognición espacial se podría ayudar al diseño espacial de los equipamientos urbanos o los espacios públicos de las ciudades, aunque resulta prematuro especular sobre estas posibles aplicaciones.

Para los propósitos del estudio de este artículo es necesario destacar los trabajos sobre la ubicación espacial o búsqueda de ruta (Lynch, 1960; Carpman \& Grant, 2002) en los que se describe el concepto de legibilidad a partir de los elementos del entorno construido que permiten navegar con éxito a través de espacios complejos como son las ciudades. La búsqueda de ruta explora la organización y la comunicación de la relación dinámica con el espacio y el medio ambiente dentro de un proceso o actividad que lleva a la movilidad-con-propósito (Passini, 1996). Lynch nombra cuatro componentes principales del proceso de la búsqueda de ruta: Orientación: ¿Dónde estoy ahora?, Decisiones de ruta: ¿Puedo encontrar el camino a donde quiero ir?, Mapeo mental: ¿Son mis experiencias consistentes y lo suficientemente comprensibles para saber dónde he estado y predecir dónde debo ir ahora?, y Cierre: ¿Puedo reconocer que he llegado al lugar correcto? La búsqueda de ruta tiene la función de informar y mostrar la información en puntos estratégicos para orientar a las personas en la dirección correcta. Según Carpman y Grant (2002), los dispositivos de orientación, tales como señales, mapas o planos de emplazamiento, se utilizan para ayudar a la gente a desarrollar un mapa mental y su plan de ruta. Este suele ser el primer nivel de la información gráfica dada para la toma de decisiones en un entorno desconocido. Los dispositivos permiten a las personas a determinar dónde se encuentran, su destino, y la mejor ruta para llegar a él por lo que la función de la señalización es la de guiarlas a lo largo de una ruta y orientarlas en la ciudad. En síntesis, la búsqueda de ruta tiene como propósito identificar cómo los individuos exploran el espacio y a partir de ello, diseñar sistemas de señalización, identificar, marcar espacios, vincularlos y organizarlos a través de medios arquitectónicos y gráficos (Galindo, 2008; Salcedo \& Caicedo, 2007).

Al analizar estas dos aproximaciones para el estudio de la ubicación espacial surgen algunas dificultades. Si bien la aproximación neurológica resulta prometedora para comprender cómo nos ubicamos en el espacio, todavía 
no conocemos el funcionamiento de estos mecanismos en los humanos y puede que nos tardemos en averiguarlo, pero aun sabiéndolo vale la pena preguntarnos: ¿qué aplicación tendría conocerlo? Aparte, claro está, de su utilidad para quienes tienen dificultades neuronales para ubicarse espacialmente. Y, la teoría del mapa mental como resultado de un proceso mental, tiene el inconveniente que los mapas dibujados por las personas a manera de esquemas no necesariamente reflejan el mecanismo que explica las relaciones que se establecen con el ambiente espacial, en la medida en que el dibujo puede estar limitado por la capacidad del individuo para representar lo que se entiende por imagen mental, y porque además, la representación que supuestamente se crea del espacio no es solo visual, razón por la cual el dibujo está limitando a lo que se pretende investigar. Algo similar sucede al pedirles a las personas que ubiquen puntos en un plano o que listen lugares; esta tarea no refleja el mecanismo que regula la conducta espacial.

Por las dificultades señaladas anteriormente, tanto en las aproximaciones neurológicas como en las cognoscitivas, se considera importante explorar desde otra perspectiva la manera como las personas se ubican en el espacio: a partir de la conducta guiada verbalmente, de la que se valen los individuos para orientar a otras con el fin de ayudarles a llegar a su destino. En consecuencia, el presente trabajo parte de la premisa de que ellas al explorar el espacio aplican reglas verbales que han aprendido siguiendo instrucciones creándolas a partir de su experiencia, las que igualmente utilizan para orientar a los demás.

\subsection{La conducta guiada por reglas}

Los humanos aprendemos a relacionarnos con el ambiente a partir de las experiencias con este, y de forma indirecta mediante reglas de las cuales las instrucciones hacen parte (Kudadjie-Gyamfi \& Rachlin, 2002). Las reglas son enunciados verbales que establecen relaciones entre el comportamiento y sus consecuencias en el ambiente, que para el caso de nuestra sociedad, se consignan en leyes, valores, consejos, o instrucciones, como el problema de que se ocupa este estudio. Y cuando estas reglas afectan o logran regular el comportamiento de quien las escucha se dice que son funcionales, en cuyo caso la regla actúa como una ocasión que establece una relación entre una forma de actuar y una consecuencia, a lo que se denomina relación de contingencia (Cerutti, 1989; Zettle \& Hayes, 1982).

Las conductas que siguen reglas se mantienen ya sea por la consecuencia social que estas producen o han generado en el pasado de la persona; por 
la sobrevaloración de sus consecuencias, como cuando se aumenta el valor motivacional por seguir o no una regla, es el caso de la publicidad; y en otras ocasiones, por las consecuencias naturales en que está organizado el ambiente psicosocial, como cuando seguimos instrucciones para armar un mueble, o cuando gracias a la regla llegamos a nuestro destino, lo cual da lugar al aprendizaje de seguir reglas en forma de instrucciones (Hayes, Zettle \& Rosenfarb, 1989; Hayes, Gifford \& Hayes, 1998; Doll, Jacobs, Sanfey \& Frank, 2009; Páramo, 2017). De tal manera que lo que va a mantener el comportamiento de seguir instrucciones será la correspondencia entre la regla y la forma en que está organizado el ambiente. Así por ejemplo cuando se dice: si usted camina cuatro cuadras en línea recta verá una escultura, estando allí, deberá girar a la derecha y caminar por una cuadra de almacenes de ropa femenina y al final de la cuadra al girar a la izquierda podrá observar al final de la calle un aviso grande con el nombre del edificio que está buscando, estableciéndose un encadenamiento entre el comportamiento del individuo apoyado en lugares, lo que se conoce como puntos de referencia, y la consecuencia final: llegar al destino. El seguimiento de esta instrucción por su parte se fundamenta en una historia de correspondencia entre la regla y las contingencias que se han organizado en el mundo socio-espacial en que se vive.

Pero las reglas no siempre se presentan de modo explícito; están implícitas, siendo posible inferir su relación propia: si hago esto, tendré como consecuencia aquello (si-entonces), de tal manera que las señales que se usan para comunicar a las personas en los espacios públicos, pueden ser vistas igualmente como reglas o instrucciones tácitas o implícitas que anuncian relaciones entre la conducta y sus consecuencias manteniendo la relación: si-entonces, como es el caso de las señales de tránsito o las señales informativas que se observan en distintos lugares.

En esta misma dirección, las expresiones corporales no vocales de las que se valen las personas para guiar a otros se constituyen en parte de la misma clase de comportamiento verbal que apoya la enunciación de la regla o instrucción en la medida en que están mediadas también por el escucha, expresiones que han sido seleccionadas en la historia personal del individuo para presentar la instrucción. De hecho el lenguaje surge y evolucionaría para referirse a acciones del individuo (Skinner, 1986).

Para el caso de esta investigación es de tener en cuenta que las señales que se observan en el espacio público establecen una regla de manera tácita. De este modo, las señales, de acuerdo con Cuesta (2010), constituyen un medio 
de comunicación visual y verbal que sirve para guiar el comportamiento, orientar el uso de un lugar y mantener prácticas sociales deseables. Sin embargo, muchos ciudadanos no logran la interpretación de estas señales o reglas expresadas mediante estímulos visuales, por lo que tratan de ubicarse y orientarse, a través de la tecnología (Internet, Waze, WhatsApp), buscando otras señales que los ayudan, o recibiendo indicaciones de otras personas para llegar a su destino cuando la señalética urbana resulta insuficiente.

El estudio se propuso explorar mediante una aproximación alternativa de los mapas mentales, de qué manera los habitantes de la ciudad se valen de un sistema de enunciados verbales, en forma de reglas o instrucciones, que les permite orientar a otros en el espacio. En lugar de estudiar la ubicación espacial mediante dibujos o preguntando directamente a los individuos sobre cómo suelen buscar rutas, aquí se enfatiza en la conducta verbal de quien da la orientación, partiendo de la suposición que al dar indicaciones a otros se evidencia de forma mucho más clara cómo las personas estructuran verbalmente su ubicación espacial en la medida en que quien da la orientación suele recurrir a una mayor especificación de las instrucciones verbales, incluyendo mayor número de puntos de referencia.

\section{MÉTODO}

\subsection{Diseño}

Estudio descriptivo de tipo exploratorio.

\subsection{Participantes}

Con el fin de explorar la conducta verbal y no vocal de las personas a partir de indicaciones de las que se sirven para orientar a otros en el espacio urbano se seleccionaron de forma aleatoria 75 personas, mayores de 18 años, hombres y mujeres, aparentemente de distintas condiciones socioeconómicas y ocupaciones, entre las que fue posible identificar: vendedores ambulantes, deportistas, policías, estudiantes y trabajadores de oficina. El estudio se ajustó a las recomendaciones éticas del gobierno colombiano en su Resolución 8430 de 1993 en lo que respecta a los artículos 6 y 11 en particular, en donde se establece en este último, en su apartado: "a", que una condición de toma de datos como la de este estudio se considera "Investigación sin riesgo" y por tanto, el investigador podrá dispensarse de la obtención del consentimiento informado (Ministerio de Salud, 1993). 


\subsubsection{Lugares}

Los participantes fueron entrevistados en cinco sitios de la ciudad de Bogotá (15 personas en cada lugar), a quienes se les indagó acerca de cómo se podría llegar desde cada uno de estos, a un lugar de destino. Los lugares para realizar las entrevistas se seleccionaron por considerarse referentes o hitos que hacen parte de la cultura urbana de los bogotanos a los que concurren gran cantidad de personas, y con los cuales se logró abarcar distintos puntos geográficos de esta ciudad. La información fue recogida en los lugares que se mencionan en la Tabla 1.

Tabla 1

Lugares de las entrevistas y lugares de destino de los entrevistados

\begin{tabular}{cc}
\hline LUGAR EN EL QUE SE REALIZó LA ENTREVISTA & LUGAR DE DESTINO POR EL CUAL SE PREGUNTABA AL \\
ENTREVISTADO
\end{tabular}

Nota: Lugares en los que se llevaron a cabo las entrevistas y al frente de cada uno de estos, el lugar de destino por el cual se preguntaba.

\subsection{Instrumento}

Para efectos de recoger la información cualitativa relacionada con la manera como las personas se ubican espacialmente a partir de las instrucciones o reglas que dan a otras se definió como técnica de recolección de información la entrevista informal.

La entrevista iniciaba con la siguiente pregunta principal: Usted me puede indicar, ¿cómo hago para llegar a... (lugar de destino)? Esta pregunta fue validada a partir de prueba piloto en algunas calles de la ciudad con población afín a la que integró el estudio definitivo. Las entrevistas informales resultado de este proceso de validación fueron llevadas a cabo por estudiantes universitarios a una muestra aleatoria de 15 personas aproximadamente en cada lugar.

\subsection{Procedimiento}

Previo a la recolección de datos se realizó un pilotaje con personas conocidas lo que permitió ajustar la pregunta orientadora, identificar algunos de los lugares en los cuales se realizaron las entrevistas a la vez que los estudiantes que tomarían los datos recibían capacitación sobre cómo formular la 
pregunta, grabar la información de manera subrepticia y registrar la información no vocal después de cada entrevista. Las entrevistas se llevaron a cabo en distintas jornadas del día, entre semana y durante los fines de semana. Estas se registraron con apoyo de una grabadora oculta para evidenciar algunos comentarios del entrevistador inmediatamente después a la entrevista, acerca de las expresiones no vocales del entrevistado entre las que se incluyeron sus gestos, la postura, la expresión facial y el contacto visual en el momento que recibía la instrucción.

Con el fin de garantizar una información espontánea no se pidió consentimiento informado teniendo en cuenta además que lo solicitado no comprometía datos personales del participante, ni implicaba daño alguno por su participación.

La información recolectada buscó establecer que las indicaciones sobre los sitios de llegada de los entrevistados respondieran a un sistema de orientación espacial. La información fue procesada mediante el programa para análisis cualitativo Atlas.ti7.0 para llevar a cabo el análisis inductivo.

\subsection{Analisis de datos}

La información recolectada fue analizada mediante el programa computarizado para análisis de datos cualitativos Atlas.ti en su versión 7.0.

\subsection{Conflicto de interés}

En la medida en que el estudio no beneficia en particular a ningún actor sino a la sociedad, los autores declaran que en el desarrollo del trabajo no se presentó ningún conflicto de intereses.

\section{RESULTADOS}

Las entrevistas exploraron la manera como las personas orientan a otras para llegar a un lugar de destino. Aunque se contactaron varias personas, algunas de ellas evitaron el contacto inicial o afirmaron no conocer el lugar que se indagaba. Se consideró apropiado un número total de 75 . No fue necesario aumentar este número en la medida en que aproximadamente después de 50 participantes la información suministrada ya era similar, lo que permitió aplicar el principio de saturación. No obstante, se decidió aumentar en cinco el número de entrevistas en cada lugar para garantizar la obtención de instrucciones o indicaciones lo más completas posible. 
Las entrevistas, junto con los comentarios sobre expresiones no vocales de parte de los entrevistadores, fueron transcritas y analizadas a partir de la creación de códigos, que en el lenguaje técnico del Atlas.ti 7.0, se refiere a las conceptualizaciones que crea el investigador sobre el material que se está analizando, que se constituyen en la unidad básica de análisis, en este caso, de las verbalizaciones de los entrevistados. Desde el inicio de la codificación se da lugar a categorizaciones más complejas, conocidas como familias dentro del Atlas.ti, las cuales agrupan códigos a partir de su correspondencia en una red semántica. Con la creación de códigos el investigador busca "atrapar" conceptualmente los comentarios de los participantes.

Partiendo de la asignación de códigos como parte del análisis de los textos se dio lugar a cinco grandes categorías que agruparon el tipo de indicaciones dadas por los participantes del estudio: instrucciones a partir de aspectos geográficos, instrucciones de una secuencia de lugares, instrucciones para reconocimiento del lugar, instrucciones tempo-espaciales y reglas no vocales, las cuales hacen parte del comportamiento verbal pero que no se manifiestan oralmente.

Se presentan a continuación los principales hallazgos desde las mencionadas categorías de análisis citando textualmente algunas de las indicaciones que dieron los participantes para dar soporte a la categoría propuesta:

\subsection{Instrucciones a partir de aspectos geográficos}

A partir de las indicaciones o instrucciones que se recibieron de los entrevistados para ir a los lugares de destino se hizo una primera agrupación de la información en códigos ${ }^{1}$ que se refieren a aspectos geográficos que el entrevistador encontraría de manera secuencial al desplazarse a pie para llegar a estos lugares, con lo cual se configura una primera categoría que se refiere a instrucciones alusivas a una secuencia geográfica. Los códigos mediante los cuales se agrupan las respuestas que indican desde una secuencia geográfica por donde las personas tendrían que caminar son: cerro, eje, esquina, bajar y subir, plano, cuadra, distancia.

Hicieron parte de esta secuencia geográfica las indicaciones que se refieren a orientaciones del tipo: siga derecho por donde es plano, sin bajar ni subir:

1 La acepción del término código en este trabajo es la de un concepto que se aplica a un fragmento del texto que se está analizando. Distinto a la acepción de la lingüística desde la cual se entiende como el conjunto de signos y símbolos de la misma naturaleza que siguen una estructura. 
Mire, váyase por aquí y siga derecho, derecho, sin bajar ni subir, por donde es plano, y donde encuentre una pendiente que es la 63, baja y ahí está Lourdes, váyase en línea recta por toda la Séptima.

Mira hacia el norte, las calles van aumentando,.... es que uno debe tener un poquito de lógica, hacia allá las calles van aumentando, esta es la 39, usted sigue derecho hasta la 63 por toda la Séptima, por el plano.

Hacen parte de esta misma categoría, las indicaciones que incluyen la geografía de las calles y algunos hitos geográficos:

Siga y siga, y allí va a encontrar usted una subida que es la circunvalar, siga por esa y ya encuentra el cerro de Monserrate allí, es una subida, no más.

Otra forma mediante la cual se valen las personas para orientar a los demás en el marco de la categoría secuencia geográfica implican indicaciones de bajar y subir:

Como está ubicado acá en el Planetario, baja acá a la carrera Séptima, toma hacia el sur derecho, derecho, derecho y llega a la Plaza de Bolívar.

Toma por la Boyacá hacia el norte hasta la calle 127 y sube por ella hasta la avenida Suba donde encuentre una subida o inclinación.

Puede subir aquí derecho, derecho, cuando llegue a la 19 aquí va mirando el número de las calles, mira allá dice 12 , ¿̇i ve?, cuando llegue al número 19 voltee a mano derecha y ahí sigue derecho.

De igual manera, hace parte de la orientación geográfica tener en cuenta la esquina y la cuadra:

Se va de aquí a la estación del Museo del Oro, o sea a la avenida Jiménez, estamos en la 11 a la 13, donde queda la iglesia o el Banco de la República, en la esquina y sube toda la avenida Jiménez hasta arriba donde diga Monserrate.

Las orientaciones que hacen parte de la ubicación geográfica se acompañan de indicaciones para que se dirijan por el norte, sur, oriente y occidente de la ciudad: 
Toma por la Boyacá hacia el norte hasta la calle 127 y sube por ella hasta la avenida Suba.

Se va por toda la vía oriente, por todo el andén oriental pasa la Universidad Javeriana, pasa unos apartamentos, hay qué más, hay por ahí... el centro cancerológico que queda en la 57, camina hacia el norte otras cuadritas y debe bajar en la 63, baja hacia el occidente 2, 3 cuadras y ya llega al parque.

\subsection{Instrucciones de una secuencia de lugares}

A partir de las instrucciones suministradas para llegar a los lugares de destino surgió otra agrupación de códigos que se refería a una secuencia de lugares, mencionados como referentes de orientación, que les indicaría a las personas que han tomado el trayecto correcto. Los códigos que lograron agrupar las instrucciones que dieron los participantes para guiar al entrevistador a partir de algunos lugares que encontrarían secuencialmente durante el recorrido se definieron como: almacenes, parques, iglesias, plazas, puentes, avenidas, edificios, señales de tránsito, como se puede observar a continuación parten de algunas de las respuestas de los entrevistados.

Conforman un grupo de lugares que encontrarían mientras se dirigen al lugar de destino: parques, plazoletas, estaciones de Transmilenio, estaciones de policía, edificaciones históricas e iglesias.

Primero, aquí en la 20 hay un parquecito que ese es el de las Nieves, sigue derecho la Plaza de Bolívar.

Pues la plazoleta del Rosario, el eje ambiental, la estatua de Simón Bolívar y la estación de policía.

Para irse por el Banco de la República y luego va subiendo y llega al famoso parque de los Periodistas, esa es la misma ruta de Transmilenio, un poquito es más se puede ir por la ruta de Transmilenio, llega hasta la calle 19 con tercera y luego Transmilenio sigue hacia el norte, entonces ustedes suben un poquito, hay otra iglesia también pequeña y sigue subiendo por ahí y llegan a la Quinta de Bolívar y sigue subiendo siga unas cuadritas donde queda City TV, de ahí sube derecho sin parar y cuando va subiendo va cogiendo así sin parar la vía de Transmilenio, sin parar va a ver una iglesia pequeñita muy antigua al lado derecho, ahí como una cuadrita coge, no se vaya a meter ni hacia abajo, ni hacia el norte, siga esa ruta y cuando esté ahí pregunte si es la Quinta de Bolívar. 
Un segundo grupo es el que referencian colegios y clubes sociales como parte de la secuencia de lugares:

Si claro, mire está aquí en la Boyacá y coge un bus que diga... que vaya por la 127. También se puede ir, va directo por toda la Boyacá, camina y encuentra la calle 116 que ahí está el colegio Segovia, que es importante, sigue caminando hacia el norte encuentra el club de Los Lagartos y hay puede voltear a mano derecha más o menos 10 o 12 cuadras hacia el oriente y diagonal encuentra el centro comercial.

Un tercer grupo corresponde al de los puentes, avenidas, estaciones de gasolina, centros comerciales, interactivos y establecimientos de entrega de mercancías:

A mano derecha por toda la Boyacá encuentra una estación de servicio Tertel, continúa hacia el norte está el liceo Segovia al frente de la calle 116, luego está el club de Los Lagartos a mano izquierda sobre la 127, sobre la 127 hay un edificio alto sobre el cerro y esa es la 127, por ahí voltea a mano derecha ahí encuentra mucha venta de automóviles y un $\mathrm{BCH}$ de entrega de mercancía, no recuerdo más.

\subsection{Instrucciones para reconocimiento del lugar}

Una tercera categoría de instrucciones que igualmente se agruparon en códigos, tiene que ver con las propiedades del lugar de destino, según las cuales se podría reconocer a partir de las características de los lugares que permitirían que las personas los reconozcan al desplazarse a pie. Los códigos que lograron agrupar las respuestas dieron lugar a descifrar aspectos con los que las personas reconocerían el lugar de destino mientras caminan en dirección a este, los cuales se definieron como: estética del lugar, dimensión, inseguridad, propiedades físicas, sitios aledaños, animales-palomas, y aglomeración de personas. A continuación se puede observar el soporte empírico de estos códigos en algunas de las respuestas de los entrevistados.

Las personas reconocen el lugar por su estética al referirse a sus formas, porque es bonito, antiguo, de gran dimensión y por su relación con el contexto:

Es un parque se ve mucha gente, mucho comercio, está la iglesia de Lourdes que es tipo como barroco, tiene una estructura muy bonita, en este momento yo creo que está en reparación, la están arreglando y hay también un centro comercial que se llama Cosmos, 
hay droguerías, hay una panadería muy antigua de bizcochos que no recuerdo el nombre, bajando a mano izquierda.

Bulevar es un centro comercial grande de dos o tres pisos, tiene arriba como una cúpula y está en vidrio por fuera al exterior.

Porque Maloka tiene dos "ula-ula" así girando, porque Maloka es una esfera.

De igual forma destacan las propiedades físicas y sus dimensiones con lo que se refieren a que es un lugar grande y amplio:

Es muy fácil de reconocer porque es una plaza grande y tiene un domo, es el cine domo, se ve el domo así grande y queda al costado derecho pero muy pegadito sobre la avenida.

Bulevar es un centro comercial grande de dos o tres pisos, tiene arriba como una cúpula y está en vidrio por fuera al exterior.

Porque usted llega y encuentra un espacio así grandísimo, el Capitolio, allá hay unos edificios así como grandes, eso es lo que se ve.

De igual manera se menciona además de la existencia de edificaciones emblemáticas la presencia de animales, entre los que se encuentran las palomas especialmente las que están en las plazas y cerca a estas edificaciones:

La Plaza de Bolívar la reconoce porque es una plaza muy grande, que está rodeada por edificios antiguos como la Catedral, el Palacio de Justicia, el Palacio de Liévano que es la casa del Alcalde y además es muy grande en el centro tiene una estatua del libertador Simón Bolívar, además tiene muchas palomas.

La aglomeración de las personas es otra de las características por las cuales se reconocería el lugar de destino. Sobre esto los entrevistados mencionaron aspectos sobre la ocupación de los vendedores ambulantes:

Reconocerá el lugar porque hay muchos vendedores ambulantes y un portal con escaleras y por ahí sube, con esto, usted sabe que ya está cerca de llegar.

\subsection{Instrucciones temporo-espaciales}

En las instrucciones que se dan a las personas para dirigir a otros a los lugares de destino se crearon igualmente códigos para agrupar instruc- 
ciones que se referían a la cercanía o lejanía que consideran tendría el lugar de destino desde donde se realizó la entrevista en relación con el tiempo que una persona necesitaría para llegar a pie. Los códigos que lograron agrupar las instrucciones dadas por los entrevistados dieron lugar a la tercera categoría conformada por los siguientes códigos: lejos, cerca, minutos, horas, kilómetros, rápido y lento. El soporte a estos códigos muestra a continuación algunas de las indicaciones dadas por los participantes.

En relación a lo cerca o lejos que puede estar el lugar de destino, los entrevistados mencionaron lo siguiente:

Realmente es cerca, tienes que caminar, ahí hay una avenida principal, ahí está igual el puente peatonal y lo cruzas y ahí sigues caminando.

Pero queda súper lejos, tiene que coger por toda esta. Por ahí una hora.

Para mí queda bastante lejos, pero si camino rápido no me demoro tanto. Por ahí media hora.

Acerca del tiempo o la distancia del recorrido, la cual puede cambiar cuando las personas lo realizan a paso, mencionaron aspectos que se relacionan con sentir que el recorrido es rápido o lento:

Por ahí unos 15 a 20 minutos a buen ritmo.

Caminando yo creo que unos 25 o media hora, pero si va a prisa pues menos de 15 minutos.

Caminando a paso así suave por ahí 20 minutos, rápido en menos de 15 minutos, es que cruzas el puente y ya ves ahí el centro comercial que dice Salitre Plaza.

Por ahí unos 20 minutos yendo a paso rápido.

De acuerdo a la distancia que las personas entrevistadas calculan para dar orientaciones mencionan el tiempo en términos de minutos y horasde acuerdo al modo de transporte que ocupan:

En bus yo creo que unos 12 o 15 minutos y a pie yo creo que unos 35 minutos

Por ahí 20 minutos a pie.

Por ahí unos 40 minutos a pie. 
Si se va en carro 5 a 7 minutos sino hay trancón, si se va en buseta igual 10 minutos.

Una horita y media.

Bulevar Niza, necesitas un bus por Suba o Transmilenio también te sirve pero te demoras más.

\subsection{Componentes no vocales de las reglas}

Las conductas no vocales estuvieron presentes en las indicaciones que los participantes dieron para llegar a los lugares de destino, las cuales permitieron configurar una quinta categoría a partir de las expresiones de los entrevistados en las que involucran los sentidos de la vista, el tacto, y en general todo tipo de conducta humana que transmite algún tipo de información mientras se daba la indicación. Estas conductas fue posible agruparlas en los siguientes códigos: gestos, movimientos corporales y posicionamiento frente al espacio.

En relación con el movimiento de las diferentes partes del cuerpo, es decir: gestos corporales, expresiones faciales, etc., se observó lo siguiente a partir de las notas que tomaron independientemente los entrevistadores:

- Algunos de los entrevistados se rascaron la cabeza o colocaron su índice en la boca con la intención de pensar hacia donde indicarnos el camino a seguir o porque no sabían cómo indicar la ruta.

- La mayoría de las personas entrevistadas, en especial los hombres, mostraron mayor cordialidad y disponibilidad a ayudar, se veían tranquilos y se acercaron al entrevistador sin mayor dificultad, mientras que las mujeres fueron más desconfiadas e indiferentes. Las personas demostraban miedo si uno se le acercaba y más si se tenía algún elemento en la mano preferían alejarse y decir que no sabían. Algunas de las mujeres que colaboraron lo hicieron con recelo, fueron las personas mayores quienes se mostraron más confiadas y amables.

- La mayoría de los entrevistados mostraron una sonrisa en su cara y gestos de amabilidad.

- En gran medida los entrevistados realizaron gestos con todo su cuerpo, dirigiendo la mirada e indicando con sus manos la ruta a seguir, direccionalidad y orientación: suba, baje, coja a la izquierda, derecha, norte y sur. 
- En la mayoría de las personas, la cara, los gestos y la vestimenta fue una barrera para realizar la entrevista a algunas personas, debido a que con el acercamiento corporal hacían gestos con su cara o eran indiferentes.

- Con varios entrevistados se establecieron contactos visuales al contactarlos para la entrevista y durante la entrevista.

En lo que tiene que ver con el posicionamiento frente al espacio, se observó lo siguiente:

- Los entrevistados, en su mayoría trataron de orientar al entrevistador de la mejor manera. Según sus conocimientos, sabían orientarse hacia arriba y abajo, al igual que el norte, sur, oriente y occidente, los más jóvenes se confundieron un poco.

- Con su cuerpo se posicionaban frente al espacio, giraban, dirigían la mirada y con sus manos la mayoría mostraban el camino a seguir y orientaban con los elementos que se encontraran a su disposición como edificios, árboles, puentes, etc.: vaya hasta el puente y gire a la derecha.

- Algunos permitían un mayor la proximidad física en la interacción, acompañamiento en el recorrido, pero no existió en ningún momento algún contacto personal.

\section{CONCLUSIONES}

La manera como las diferentes teorías se han aproximado a explicar la cognición espacial y particularmente la búsqueda de rutas o los procesos involucrados en la movilidad-con-propósito, de un origen a un destino, ha sido a través de los mapas mentales (Allen, 2003; Devlin, 2012), o mediante modelos que buscan comprender la organización del conocimiento derivado de la exploración del espacio (Grasser \& Goodman, 1985). Pero más recientemente, con el auge de las ciencia cognitiva, y la neurociencia la aproximación teórica ha sido desde las tecnologías fMRIs y PET para ubicar zonas del cerebro asociadas a la ubicación espacial y búsqueda de rutas, y desde el conexionismo, basado en redes neuronales artificiales (Hanson \& Burr, 1990; Campanario, 2004) con las que pretenden emular el funcionamiento de las redes neuronales del cerebro humano mediante programas de cómputo para explicar el funcionamiento neuronal manipulando fuentes de estimulación externa al modelo que se crea, y así establecer el efecto de un elemento del ambiente en una actuación o representación cognoscitiva que pueda crear el individuo sobre este. Para el caso de la tarea de búsqueda de ruta 
se han propuesto igualmente modelos de mapas cognoscitivos neuronales (Levenick, 2007; Wallgrün, 2010) e incluso para tratar de explicar lo que en este trabajo se ha mostrado como resultado del seguimiento de reglas verbales, buscando establecer los mecanismos cognoscitivos que fundamentan las direcciones dadas verbalmente y la toma de decisiones frente al espacio (Couclelis, 1996; Allen, 2003). Para los proponentes de estos modelos, el lenguaje refleja la manera como las personas piensan acerca del espacio, lo enfrentan y lo construyen en la mente. Sin embargo, al igual que lo menciona Couclelis (1996), la relación entre las palabras expresadas y las estructuras cognitivas que las subyacen está lejos de ser transparente, mucho más cuando se trata de entender cómo una breve descripción acerca de un ambiente desconocido para otra persona se intenta incorporar en esa otra mente. La manera más eficaz, como lo proponemos en este artículo, es estudiando directamente el lenguaje y su estructura funcional, no lingüística, en la orientación de rutas, mas no como una representación de un mapa mental interno, en la medida en que de esta manera no se pueden hacer accesibles de forma directa las variables a estudiar, lo que no sucede con las reglas verbales de fácil acceso.

No sabemos todavía cómo se codifican en nuestro cerebro algunas características espaciales que permiten organizar el mapa neurológico a la manera como lo establecieron John O'Keefe y los esposos May-Britt y Edward Moser en animales. Mientras tanto, se puede afirmar que en términos del lenguaje las personas estructuran su conocimiento del espacio urbano a partir de un sistema de categorías complejas que tienen que ver con el seguimiento de reglas que a la vez se reflejan en instrucciones o comunicación no vocal o gestual a manera de expresiones corporales cuando tienen que orientar a otros. Estas categorías verbales se estructuran alrededor de la ubicación geográfica y del reconocimiento de lugares, de instrucciones para reconocimiento del lugar, temporo-espaciales y manifestaciones no vocales, todas ellas aprendidas con seguridad, a partir de una relación funcional con el entorno.

La identificación de estos elementos que conforman una estructura verbal, al menos de manera exploratoria como se ha hecho en este trabajo, puede arrojar pistas sobre la organización de la información espacial en el cerebro de los humanos, pero como se mencionó anteriormente, estamos todavía lejos de establecer estos mapas neurológicos, más aún si se trata de encontrar su relación con el lenguaje. 
Por ahora basta con mostrar la evidencia que permite establecer al menos de manera preliminar las reglas básicas que reflejan la estructura verbal para orientarse en el espacio, lo que da lugar a hipotetizar una gramática espacial a la que subyace una sintaxis o conjunto de reglas que mostraría una conectividad entre espacios y sus propiedades junto con nuestras nociones de tiempo, conectadas por el lenguaje verbal y no vocal, sostenida esta sintaxis por las consecuencias naturales a que conduce el seguimiento de instrucciones.

En consecuencia, no es suficiente con suponer la existencia de un mapa mental para explicar la movilidad-con-propósito, sino que es indispensable explorar los mecanismos de aprendizaje que explican dichos procesos, que para el caso, correspondería a la forma como está estructurado funcionalmente el lenguaje (Hayes, Gifford \& Hayes, 1998; Costa, Pereira \& Lovo, 2017), su relación con el espacio y las contingencias naturales para el seguimiento de instrucciones, por lo que la conducta espacial guiada por reglas podría ser una alternativa prometedora. Será necesario, por consiguiente, seguir explorando de manera más profunda cómo se estructura el comportamiento verbal del que se valen las personas para explorar el espacio y orientar a otras en cuanto tratan de ubicarse espacialmente.

Finalmente se mencionan algunas implicaciones de tipo educativo y de planeación urbana derivadas del estudio. En relación con la educación, estos hallazgos pueden tener utilidad tanto en los programas de formación de maestros como para la enseñanza de la exploración de la ciudad que hacen los niños, en la medida en que se incorporen las categorías identificadas en el estudio para la orientación espacial de forma más precisa, a la manera de una gramática espacial desde la enseñanza de la geografía y en la forma como a los niños se les enseña a relacionarse con la ciudad. Respecto de la planeación urbana podría igualmente sugerirse que la ciudad deberá ser más diversa en su diseño espacial para efectos de conseguir una mejor orientación o ubicación por parte de sus habitantes y visitantes. Sin lugar a dudas una clara nomenclatura contribuye de forma significativa para orientarse por la ciudad si se compara con aquellas ciudades donde predominan nombres de calles como puntos principales de referencia. Una clara organización de la nomenclatura se constituye en un elemento clave para que al seguir instrucciones de orientación se consigan los resultados esperados, llegar al lugar deseado. La monotonía del diseño espacial de las ciudades en cuanto a sus parques, avenidas y arquitectura no contribuye a la estimulación visual y a la diversidad necesaria para tener mayores puntos de referencia que permitan 
encadenar el seguimiento de instrucciones, lo cual se refleja en las pocas menciones en el estudio a puntos de referencia destacados en la ciudad. Así entonces, consideramos que además de la necesidad de contar con una clara nomenclatura y un diseño urbano diverso, se requiere de la implantación de un sistema de señalética urbana moderna que deberá, junto con los mapas de movilidad, ubicarse en puntos de interconexión de lugares y hacer referencia a lugares visibles de la ciudad y reconocidos por sus propiedades espaciales.

Financiamiento: El estudio es resultado del proyecto de investigación titulado Gramática Espacial Urbana, financiado por la Universidad Pedagógica Nacional (DED-369-14).

Agradecimientos: Se agradece la participación de María Helena Díaz, estudiante de la Maestría en Educación de la Universidad Pedagógica Nacional en el proceso de recolección de datos. La estudiante en mención junto con: Alejandra Ponce de León, Martha Irene Guzmán y Luis Alberto Reyes, hicieron parte del equipo de investigación para llevar a cabo el trabajo de campo del proyecto institucional de la de la Universidad Pedagógica Nacional: Gramática Espacial Urbana.

\section{REFERENCIAS}

Allen, G.L. (2003). Gestures accompanyng verbal route directions: Do they point to a new avenue for examining spatial representations? Spatial Cognition and Computation, 3, 259-268. http://psycnet.apa.org/doi/10.1207/s15427633scc0304_1

Anooshian, L. J., \& Young, D (1981). Developmental changes in cognitive maps of a familiar neighborhood. Child Development, 52(1), 341-348. http://psycnet.apa. org/doi/10.2307/1129248

Bronzaft, A.L., Dobrow, S.B., \& O'Hanlon, T.J. (1976). Spatial orientation in a subway system. Environment and Behavior, 8, 575-594. http://psycnet.apa.org/ doi/10.1177/001391657684005

Campanario, J. M (2004). El enfoque conexionista en psicología cognitiva y algunas aplicaciones sencillas en didáctica de las ciencias. Enseñanza de las Ciencias, 22(1), pp.93-104. https://www.raco.cat/index.php/ensenanza/article/ viewFile/21963/21797

Carpman, J., \& Grant, M.A. (2002). Wayfinding: A borad view. In R. Bechtel \& A. Churchman; Handbook of Environmental Psychology. New York. Wiley.

Cerutti, D. T. (1989). Discrimination theory of rule-governed behavior. Journal of the Experimental Analysis of Behavior, 51(2), 259-276. https://dx.doi.org/10.1901\%2Fjeab.1989.51-259

Costa, M.M., Pereira, F., \& Lovo, L.A. (2017). Higher-order verbal behavior: theoretical-empirical analysis of autoclitic effects on on-verbal behavior. In J.C. Todorov (Editor), Trends in Behavior Analysis (vol 2). Brasilia: Technopolitik.

Couclelis, H. (1996). Verbal Directions for Way-Finding: Space, Cognition, and Language. In: Portugali J. (eds), The Construction of Cognitive Maps. GeoJournal Library. Dordrecht: Springer.

Cuesta, O. (2010). Señalización educativa para la convivencia en el espacio público. Signo y Pensamiento, 29(57), 458-470. http://revistas.javeriana.edu.co/index. php/signoypensamiento/article/view/2535 
De Alba, M. (2004). Mapas cognitivos: historia de un concepto y sus aplicaciones al análisis del espacio. En S. Arciga (Edit.), Del pensamiento social a la participación. Estudios de psicología social. México: SOMEPSO. Sociedad Mexicana de Psicología Social.

Devlin, A.S. (2001). Mind and maze: Spatial cognition and environmental behavior. Westport, CT: Praeger. http://dx.doi.org/10.1002/acp.938

Devlin, A.S. (2012). Environmental Perception: Wayfinding and Spatial Cognition. In S.D. Clayton, The Oxford Handbook of Environmental and Conservation Psychology. New York: Oxford University Press. http://www.oxfordhandbooks.com/view/10.1093/oxfordhb/9780199733026.001.0001/oxfordhb-9780199733026-e-3

Doll, B.B., Jacobs, W.J., Sanfey, A.G., \& Frank, M.J. (2009). Instructional control of reinforcement learning: a behavioral and neurocomputational investigation. Brain Research, 1299, 74-94 https://www.ncbi.nlm.nih.gov/pubmed/19595993

Downs, R., \& Stea, D. (2005). Image and Environment: Cognitive Mapping and Spatial Behavior. Chicago: Aldine Transaction. https://doi.org/10.1080/00690805.1976. 10437937

Galindo, J. (2008). Comunicación, ciencia e historia. New York: McGraw-Hill.

Grasser, A.C., \& Goodman, S.M. (1985). Implicit knowledge, question answering and the representation of expository text. In B.K. Britton \& J.B. Black (eds.), Understanding Expository Texts. Hillsdale, Nueva Jersey: Lawrence Erlbaum.

Hafting, T., Fyhn, M., Molden, S., Moser, M.B., \& Moser, E.I. (2005).Micro structure of spatial map in the entorhinal cortex. Nature, 436, 801-806. http://doi. org/10.1038/nature03721

Hanson, S.J. \& Burr, D.J. (1990). What connectionist models learn: Learning and representation in connectionist networks. Behavioral and Brain Sciences, 13, 471518. http://psycnet.apa.org/doi/10.1017/S0140525X00079760

Hayes, S. C., Zettle, R. D., \& Rosenfarb, I. (1989). Rule following. In S. C. Hayes (Ed.), Rule-governed behavior: Cognition, contingencies, and instructional control (pp.191-220). New York: Plenum.

Hayes, S.C., Gifford, E.V., \& Hayes, G.J. (1998). Moral behavior and the development of Verbal regulation. The Behavior Analyst, 21(2), 253-279. https://doi. org/10.1007/BF03391967

Holding, C. S. (1992). Clusters of reference points in cognitive representations of the environment. Journal of Environmental Psychology, 12(1), 45-55. http://psycnet. apa.org/doi/10.1016/S0272-4944(05)80296-8

Kaplan, S. (1973). Cognitive maps in perception and thought. In R.M. Downs \& D. Stea (Eds.), Image and environment: Cognitive mapping and spatial behavior (pp. 63-78). Chicago: Aldine. https://doi.org/10.1080/00690805.1976.10437937

Kenneth J., Lohmann, M. F., Lohmann, N., Putman, F. (2007). Magnetic maps in animals: nature's GPS. Journal of Experimental Biology, 210(21). http://jeb.biologists.org/content/210/21/3697

Kirasic, K.C., Allen, G.L., \& Siegel, A.W. (1984). Expression of configurational knowledge of large-scale environments: Students performance of cognitive tasks. Environment and Behavior, 16(6), 687-712. http://psycnet.apa.org/ doi/10.1177/0013916584166002

Kitchin, R., \& Blades, M. (2002).The cognition of geographic space. Londres: I.B. Tauris.

Kudadjie-Gyamfi, M., \& Rachlin, H. (2002). Rule-governed versus contingency-governed behavior in a self-control task: effects of changes in contingencies. 
Behavioral Processes, 57(1), 29-35. http://dx.doi.org/10.1016/S03766357(01)00205-4

Levenick, J.R. (2007). NAPS: a Connectionist Implementation of Cognitive Maps, Connection Science, 3(2), 107-126. https://doi.org/10.1080/09540099108946580

Levin, M (1982). You-are-here maps: Psychological considerations. Environment and Behavior, 14, 221-237. https://doi.org/10.1177/0013916584142006

Lynch, K. (1960). The image of the city. Cambridge, MA: MIT Press.

Manzanero, A.L. (2008). Memoria y contexto. En A.L. Manzanero, Psicología del Testimonio (pp.59-82). Madrid: Pirámide.

Matthews, M.H. (1987). Sex differences in spatial competence: The ability of young children to map primed unfamiliar environments. Educational Psychology, 7(2), 77-90. https://doi.org/10.1080/0144341870070201

Milgram, S., \& Jodelet, D. (1976). Psychological maps of Paris. In H. Proshansky, W. H. Ittelson \& L. Rivlin (Eds.), Environmental psychology: people and their physical settings (pp.104-124). New York: Holt Rinehart and Winston.

Morrow, L., \& Ratcliff, G. (1988). Neuropsychology of spatial cognition: Evidence from cerebral lesions. In J. Stiles-Davis, M. Kritchevsky, \& U. Bellugi (Eds), Spatial cognition: Brain bases and development (pp.5-32). Hillsdale, NJ: Erlbaum.

Ministerio de Salud. República de Colombia, Resolución No. 8430, octubre 4 de 1993.

Moser, G. (2012). Cities. In S.D. Clayton, The Oxford Handbook of Environmental and Conservation Psychology. pp.2013-220. New York: Oxford University Press. http://www.oxfordhandbooks.com/view/10.1093/oxfordhb/9780199733026. 001.0001/oxfordhb-9780199733026

Morris, R.G.M. (1981). Spatial localization does not require the presence of local cues. Learning and Motivation, 12(2), 239-260. https://doi.org/10.1016/00239690(81)90020-5

Navarro, O., Lozano, N. \& Rodríguez, U. (2017). Los mapas cognoscitivos o la adquisición de un saber espacial como método de investigación social. En: P. Páramo, La investigación en ciencias sociales: la recolección de información. Bogotá: Universidad Piloto de Colombia.

O'Keefe, J. (1976). Okace units in the hippocampus of the freely moving rat. Experimental Neurology, 51(1), 78-109. https://doi.org/10.1016/0014-4886(76)90055-8

Páramo, P. (2017). Reglas Proambientales. Revista Suma Psicológica, 24(1), 42-58. https://doi.org/10.1016/j.sumpsi.2016.11.001

Passini, R. (1996). Wayfinding design: Logic, application and some thoughts on universality. Design Studies, 17(3), 319-331. https://doi.org/10.1016/0142 -694X(96)00001-4

Pezzulo, G., Donnarumma, F., \& Dindo, H. (2013) Human Sensorimotor Communication: A Theory of Signaling in Online Social Interactions. PLOS ONE, 8(11). https://doi.org/10.1371/journal.pone.0079876

Real Academia Española (2014). Diccionario de la lengua española, 23.a ed. Madrid: Espasa.

Roberts, W.A. (1984). Some issues in animal spatial memory. In H.L. Roitblat, T.G. Bever \& H.S. Terrace (Eds.), Animal Cognitio. Hillsdale, N.J: Erlbaum.

Salcedo, M. A., \& Caicedo, S. (2007). Señales visuales urbanas en algunos cruces viales de Cali, Colombia. Revista Cientifica Guillermo de Ockhan, 5(2), 11-133. http://dx.doi.org/10.21500/22563202.510 
Skinner, B.F. (1986). The evolution of verbal behavior. Journal of the Experimental Analysis of Behavior, 45(1), 115-122. https://dx.doi.org/10.1901\%2Fjeab.1986.45-115

Stea, D. (1974). Architecture in the head: Cognitive mapping. In J. Lang, C. Burnette, W. Moleski, \& D. Vachon (eds.), Designing for human behavior: Architecture and behavioral sciences. (pp.157-168). Stroudsburg: Dowden, Hutchinson y Ross Inc.

Tolman, E.C. (1948).Cognitive maps in rats and men. Psychological review, 55(4), 189-2. http://psycnet.apa.org/doi/10.1037/h0061626

Wallgrün, J.O. (2010). Qualitative spatial reasoning for topological map learning. Spatial Cognition \& Computation. An Interdisciplinary Journal, 10(4), 207-246. https://doi.org/10.1080/13875860903540906

Zettle, R. D., \& Hayes, S. C. (1982). Rule-governed behavior: A potential theoretical framework for cognitive-behavioral therapy. In P. C. Kendall (Ed.), Advances in cognitive-behavioral research and therapy (Vol. 1, pp.73-118). New York: Academic Press. 


\section{ANEXO \\ Gramática Espacial Urbana: la orientación espacial guiada por reglas verbales}

\section{ENTREVISTA}

El objetivo de la entrevista es recoger información cualitativa relacionada con la manera como las personas se ubican espacialmente a partir de las instrucciones o reglas que dan a otras personas. Para ello, los investigadores o a quienes se delegue llevan a cabo una entrevista a personas de Bogotá, en distintas jornadas del día, entre semana y durante los fines de semana.

Para este particular, el entrevistador deberá:

a. Verificar que el almacenamiento de la grabadora sea suficiente para el desarrollo de la entrevista; verificar con anterioridad el alcance para la grabación y nitidez del audio.

b. Seguir el protocolo para el inicio y cierre de la entrevista, teniendo en cuenta la informalidad de la entrevista acercándose al entrevistado de manera natural evitando el rechazo a la pregunta que se va a hacer y mostrándose muy espontáneo.

Para el desarrollo de la entrevista:

- El entrevistador realizará la siguiente pregunta.

- Introducirá la pregunta de la siguiente manera con una frase informal estando en el lugar en el que se realizará la entrevista:

Disculpe /perdón señor(a) /, señor(a) me puede indicar /por favor, me puede decir cómo hago para llegar a... (Lugar de destino)?

c. Tener en cuenta el siguiente listado de lugares para realizar la pregunta:

\begin{tabular}{cc}
\hline LUGAR EN EL QUE SE REALIZA LA ENTREVISTA & LUGAR DE DESTINO POR EL CUAL SE PREGUNTA AL ENTREVISTADO \\
\hline Planetario de Bogotá & Plaza de Bolívar \\
Centro Comercial Titán & Centro Comercial Bulevar Niza \\
Plaza de Bolívar & Monserrate \\
Centro Comercial Gran Estación & Maloka \\
Parque Nacional Enrique Olaya Herrera & Iglesia de Nuestra Señora de Lourdes \\
\hline
\end{tabular}

d. Tenga presente realizar el registro inmediatamente después a la entrevista de las expresiones no vocales del entrevistado entre las que se incluyen gestos, postura, movimientos corporales y expresión facial. 